\title{
THE EFFECT OF TIME PRESSURE ON THE PERFORMANCE OF DEXTEROUS OPERATIONS
}

\author{
M. Pooripanyakun $\bowtie$, A. Wodehouse and J. Mehnen \\ University of Strathclyde, United Kingdom \\ $\triangle$ munyaporn.pooripanyakun@strath.ac.uk
}

\begin{abstract}
This study explores the effects of time pressure in dexterous operations on two types of interface: the fixed interface and the moving interface. Results show that the accuracy of finger movement is decreased, the information processing on the sense of sequence, position and direction is worsened by the psychological disturbance. The findings indicate that a fixed interface is more robust to performance and participants can learn and perform tasks quicker than a moving interface. Finally, the researchers give some practices on both fixed and moving interface design.
\end{abstract}

Keywords: design cognition, performance indicators, design guidelines

\section{Introduction}

The product interface is the part of the product with which users see and interact. Performing tasks or interacting with products require different capacities of mental workload. Norman (1983) explained the mental model that the user formulated resulting from the user's technical experience, previous familiarity from a similar system and human information processing formation. Norman (1984) also discussed the design of products or interfaces which contributed to the human cognitive performance. Meanwhile, Csikszentmihalyi (1997) explained the different states of action related to the challenge of the task and the individual's skill. From his concept of user experience, he implied that people could encounter anxiety and stress if they perceive that the task goal exceeds their ability. The users could perceive the product interface and perform differently in each situation, for example in a severe circumstance and time-constraint condition. The emotions, intentions, and thoughts are also connected and affect one another. Under the stressful circumstance, the senses of users tend to be reduced and their reaction might be diminished (Illera et al., 2010). Negative emotions usually lead to improper operations subconsciously. Moreover, when short-term memory is overloaded, it can lead to performing a mistake (Norman, 1984). This brings about the risk of error or accidents on the user. Bakker et al. (2016) illustrated the type and quality of interaction consisting of focused interaction, peripheral interaction, and automatic system behaviour. These are parts in everyday life to interact with any devices. Both consciousness and intention also involve in human interaction.

The concepts of mental workload have been involved in many subjects of a study (Young et al., 2015). For example, in human-computer interaction and a study of situation awareness error. Rasmussen and Vicente (1989) asserted that human errors can be reduced by designing a supportive ecological interface. The cognitive workload plays an important role in the human performance. Wu et al. (2016) found that interface complexity has much impact on the operator's response. Hughes and Babski-Reeves 
(2005) also presented the effects of time pressure and mental workload on workload perceived and interaction. However, it is quite rare to discover the study of interface design in relation to details of performance, mistake pattern, error analysis and human behaviour. Many studies has been built around a single interface type and not explored finger operations in detail. The interface could generally be divided into two types: fixed and moving. The fixed interface is the part where the user interacts that is stationary. On the other hand, the moving interface is the part where the user interacts that is dynamic and movable. The new challenge in research is the interface and interaction design for performance accuracy in an increasingly complex world. Proctor and $\mathrm{Vu}$ (2016) conveyed the concept of improved interface design from psychological aspects, contributing to the experimental design in this study.

This research, therefore, has explored the effects of mental disturbance on tasks through a fixed and a moving interface to answer the question of how the interfaces affect performance accuracy under time pressure. To see how respondents deal and interact with different interfaces, the researchers have chosen a keyboard, a basic existing (fixed) interface for studying interaction. Richardson and Vecchi (2002) stated that the visuospatial task was suitable for investigating the mental pathway, human cognition, and coordination. Thus, the jigsaw piece, an interface on which a person can manipulate directly (moving), has been explored in addition to control the keyboard interface.

Two hypotheses have been put forward: the time-pressure significantly affects the performance accuracy on the fixed interface; the time-pressure significantly affects the performance accuracy on the moving interface. Performing tasks under time-pressure as well as new challenging experience has been investigated. With a conceptual model of the use of a system, Young (1983) claimed that the performance could be explained with the choice of method, details of performance, and locus and nature of errors. Therefore, interaction, errors, and patterns of a mistake have been examined on the performance of fine dexterous operations. In order to ensure that the designers will understand and create a better product interface, the research has given some design practices on both fixed and moving interfaces. Figure 1 shows the conceptual framework of this study that explored performance (net speed, error, behaviour) when pressure is applied and unapplied to participants via different interface types and interaction styles (tasks). The task on the keyboard is considered as an episodic batch action and the jigsaw task is seen as a holistic process. If the designers understand a feasible mistake pattern from the cognitive and physical aspect, they can improve the design for speed and smoothness operations prevented from an unintentional mistake.

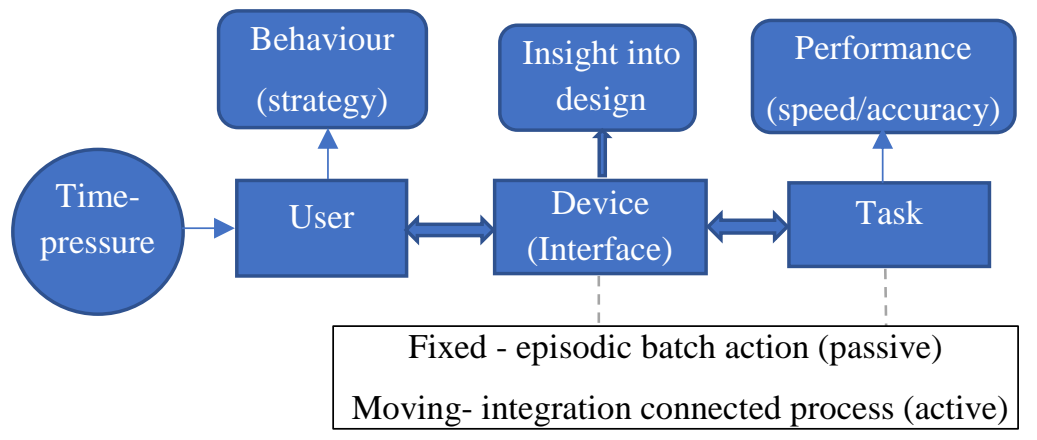

Figure 1. Conceptual framework

\section{Method}

\subsection{Design and approach}

The configuration of tasks in the experiment is shown in Figure 2. Task 1 is pressing buttons sequentially on a Standard English keyboard panel corresponding to the set of Thai words presented in the left column of the document. The letter displayed on a button is different from the letter typed in order to test the participant's cognitive mapping and finger movement on the control interface. With unfamiliarity, the non-Thai participants perceive each word as a symbol and retrieve information from the memory they just learned and practiced, then perform the corresponding procedure. The 3designated words are constructed with the position of a key to be performed in different directions 
with both hands. Each specific word needs pressing the keys in a three-sequence action. For example, press a series of ' $i$ ', ' $v$ ' and ' $p$ ' then press the space bar for one specific Thai word. The indirect strange symbols that participants see at that moment consisted of the 20 words per trial. Figure 3 presents the interaction sequence for 3 words in this task.

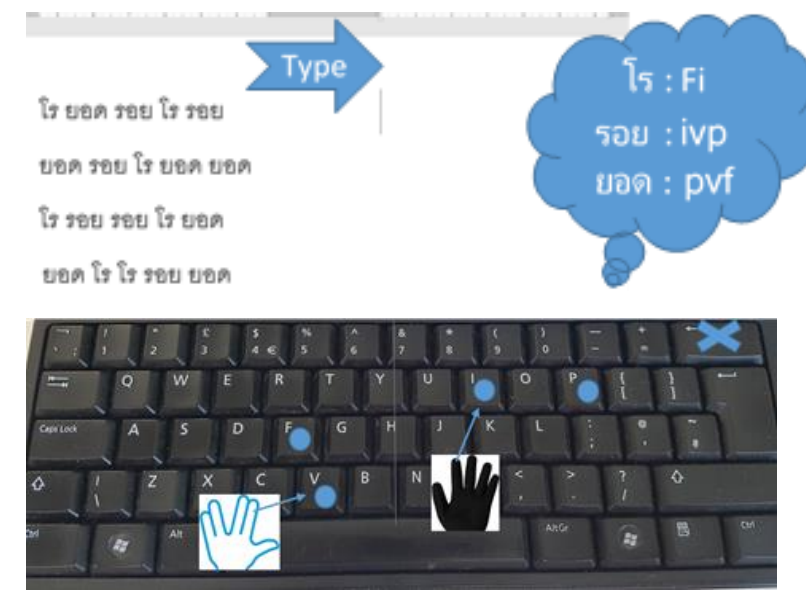

(a)
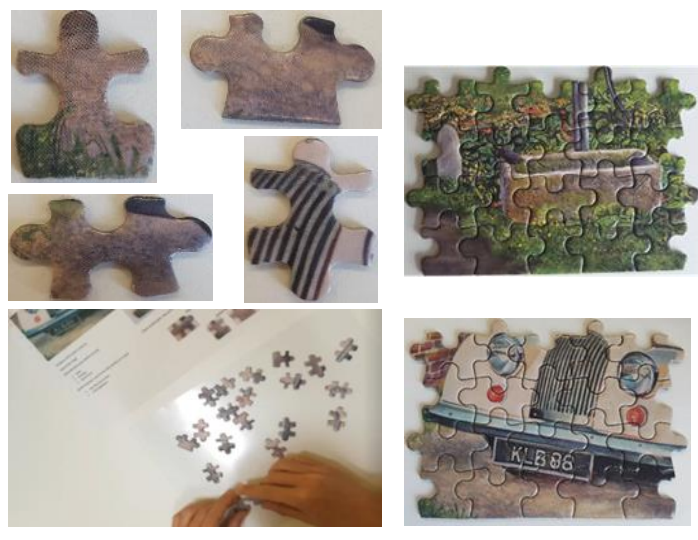

(b)

Figure 2. The designed experiment on Task 1 and Task 2

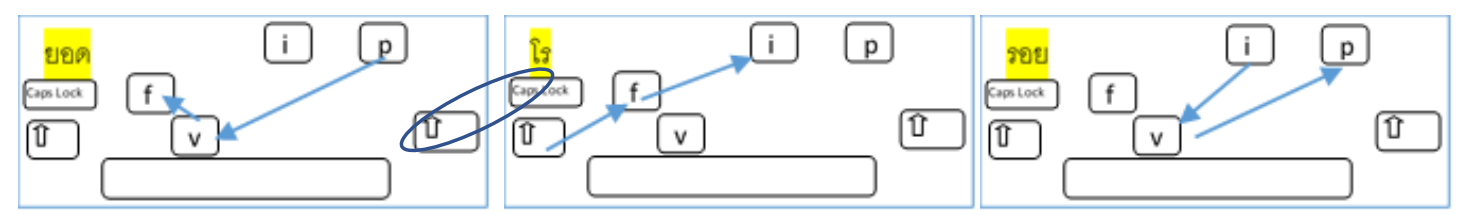

Figure 3. The design of interaction in Task 1

Task 2 is a putting a 20-piece jigsaw puzzle into place to form an image that participants saw and practised one time before the test. The 20 different jigsaw pieces are a part of a picture of 500 pieces puzzle, containing 5 bottom-flat edge pieces and 15 interior pieces. There are two shapes: vertical and horizontal which should be connected in a switching manner. Participants have to consider a lot of the information from each piece at the same time and fit the piece in the correct position and direction to construct the picture. The word and the jigsaw piece are presented in a random position on each trial. Both tasks require eye-hand coordination along with cognitive ability.

It should be noted that there are some differences between tasks. In Task 1 participants must perform the same pattern. They need to acknowledge a set of any word among 3 words on the screen and response on the keyboard with the corresponding sequence. The task duration is relatively short (less than 40 seconds) and the error is independent. Although doing a jigsaw in Task 2 is for the same picture, there is no apparent procedure. All pieces are related. A holistic viewing, careful observation, and individual strategy are a necessity. It should be consumed a lot of time and psychic energy. The spatial change of the pieces on each trial could extend the orientation time as well. Illera et al. (2010) claimed that the more information a person had, the more time and effort to process.

\subsection{Participants}

The 21 non-Thai participants ( 12 males, 9 females) consented to involve in this study. They were from an engineering and management background, aged between 23 and 36. Everyone was a right-handed person. Participants got the same protocol in performing two tasks. Most participants (13 from 21) had the ability to touch typing and everyone did a typing task regularly. Everyone in this study has experience in doing a jigsaw. Wu et al. (2016) claimed that user background had no interaction effect on the interface complexity, although it could influence the user experience. This assertion could support the study of interface complexity for this research.

Based on the normal distribution assumption, the participants in the study were randomly selected and paired to both normal and time-pressure conditions. 


\subsection{Experimental procedure}

At the beginning of each task, participants got instructions and practice time. They were free to ask the experimenter about the mission they were assigned. Participants were advised to do both tasks as quickly and correctly as they could. For Task 1, they practiced by typing each word pattern. For Task 2 , they practiced by forming the 20 pieces of jigsaw until picture completeness. The experiment consisted of two sessions: the normal session (control) and the time-pressure session (experiment). Each session was conducted in 4 trials consecutively. Trial 1 to Trial 4 was for measuring baseline efficiency and training. The instructions were still available at this stage of the experiment but had been taken out during the time constraint condition. They were not allowed to edit any mistakes while performing Task 1. However, they could see the latest outcome before starting the new trial. A stopwatch was used to measure the response time in the normal session. While the countdown timer was set at 4 levels in the pressure situation. The values were set descending on Trial 5 to trial 8 from 35, 30, 20, 15 seconds, respectively on Task 1 and from 80, 70, 60, 50 seconds, respectively on Task 2. The task target was set rigidly to challenge participants' capability. Participants had to finish 20 words and 20 pieces of the jigsaw puzzle in a limited time.

The experiment was conducted in the unobtrusive meeting room. Participants were also asked to wear a fitness tracker wrist band with a photoplethysmographic (PPG) heart rate sensor to measure a physiological response. The video recording was used to observe their interaction. A brief and informal interview was conducted before and after the experiment about their background and opinion on the task they just performed.

Unfortunately, after the experiment had been conducted ten times, the colour of jigsaw pieces faded. Thus, the experimenter had to use another puzzle set for the rest of the experiment (Participant number 11 to 21 used the jigsaw Set 2). Although the jigsaw view was different as shown in Figure 2(b) but had a similar complexity (20 pieces from the same puzzle box set). The overall experiment lasted around an hour.

\subsection{Data analysis}

The speed performance was analysed by the response time recorded, the number of words or number of jigsaw pieces done and right in each trial. The task completeness and accuracy were explored. Then the net speed, the product of speed and accuracy, was analysed. A comparison of the net speed along with the error rate was made between the normal and time-pressure conditions. The paired t-test was used for the hypothesis tests of the dependent participants under a 95\% confidence level. Moreover, the characteristics of error and pattern of a mistake were categorised and extensively investigated. Furthermore, participants' interaction and behaviour observed from the video were summarised and discussed.

\section{Result and discussion}

\subsection{Performance analysis}

Task1 The result appears that many participants could not finish the task or reach the assigned speed during the time constraint condition. There was only one person that finished the task in time in Trial 8 with a target of typing 20 words within 15 seconds or 80 words per minute (wpm) shown with the circle dot in Figure 4(a). Surprisingly, the speed appears to be an upward trend even in a difficult situation. However, the more the rapidity, the higher the number of errors. It should be noted that the average speed in the normal session (30.72 wpm) was lower than those in the timeconstraint session (39.34 wpm) and there was a significant difference among these conditions $(\mathrm{p}=$ $0.001<0.05)$. Although the standard deviation of speed overall the experiment was high about 12.4 on average, a statistical test indicated that there was no significant difference between the normal and time-pressure situations $(\mathrm{p}=0.148)$ among participants. This means that participants perform the task with consistency but have a dramatic difference in typing skills. With the fixed interface, they could use their proprioceptive sense other than the cognitive ability to facilitate the task (Probst, 2016). 


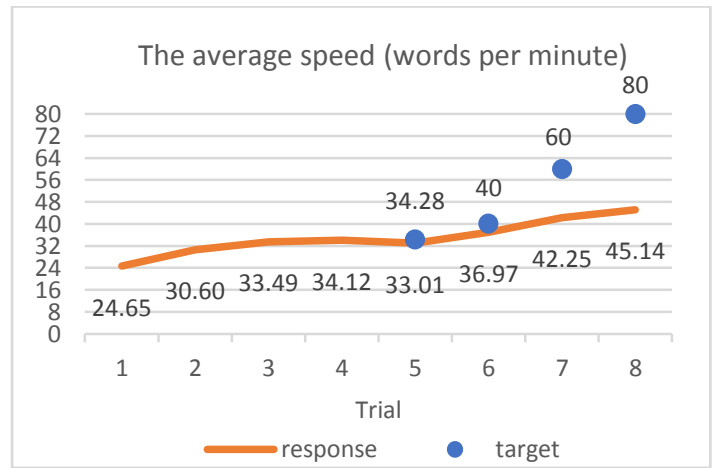

(a)



(b)

Figure 4. The speed and accuracy outcome for Task 1

Despite the trend on the graph was increasing as the learning process, the speed improvement became the pivot at Trial 5 which was the transition to the stressful situation. Figure 6 shows the average speed increment analysed from the difference between the speeds of the adjacent trial. It can be seen that the speed was dropped between Trial 4 and Trial 5 that is why the value in Figure 6(a) was negative in that period. However, a big improvement occurred after that between Trial 5 and 7 shown on the graph marked by backslash sign. To exemplify, the average of the speed increment in the normal condition was 3.15 but was unexpectedly developed to 4.04 in the stressful condition. Zeidner and Matthew (2011) suggested that on the easy task, the capability may be facilitated by anxiety.

On the other hand, Figure 4(b) presents the average error rate that occurred in each trial. It should be noted that the average error rate in the normal session (1.38 per trial) was lower than those in the time-constraint session (2.06 per trial) and was a significant difference $(p=0.04)$. It was found that after getting familiar with the task in both conditions (Trial 1 and Trial 5), the number of errors was dropped as the participants tried to correct the previous error and adapted themselves to the circumstance. However, with the more on difficulty as well as the participant expectation for the best result on the test put themselves under the pressure and leaded to the rise of mistakes again on Trial 3 to 4 and Trial 7 to 8 . Overall, the error rate tended to increase. The results were similar to Hughes and Babski-Reeves's findings (2005) that time pressure resulted in growing of typing speed and errors.

Task2 Similar to Task 1, participants could not finish the task at a given time, especially on Trial 8 . The clustering outcome patterns were turned out in the time-constraint condition. The speed performance did not meet the target speed. It should be noted that the average speed in the normal session ( 8.09 piece per minute, $\mathrm{ppm})$ was lower than those in the time-constraint session $(12.37 \mathrm{ppm})$ with a significant difference $(\mathrm{p}=0.001)$. The standard deviation value was about 2.74 on average. The performance was presented separately because of using two sets of the jigsaw (Figure 5). The average speed between the jigsaw set was significantly different $(\mathrm{p}=0.00)$. It seems that Set1 was more difficult than Set 2 . The car picture of Set 1 was shown in the inclination form not parallel to the ground and there were many subparts in the picture. In contrast, the pool picture in Set 2 was represented in a straightforward pattern of one thing. Additionally, from observation and informal interviews, it was found that the majority of participants in jigsaw set 2 appears to be the 'field independence' (FI) personality trait as most participants in this group seems to be enthusiastic in the jigsaw test. To exemplify, the FI was a characteristic of a person who had a special skill in organising, developing the information into a structure in contrast to the 'field dependence' (FD) personality (Hong et al., 2012). The difference in the participants' cognitive style and the picture difficulty led to a difference in response. However, the effect of time pressure on this task was a similar trend in both jigsaw sets.

Looking at the speed improvement in Figure 6 (b), the speed progress seems to continue as the time elapsed even in a difficult situation. However, a large increment occurred in the normal session during Trial 1 to Trial 4, in contrast with Task 1 . The possible reason for higher improvement at the beginning would be from a long learning process of the moving interface. However, after starting the time-limited session, the speed increment trend was dropped. In other words, the average of the speed 
increment in the normal condition is 1.28 but is 0.76 for the stressful condition. This indicated that the performance improvement on the moving interface seems to lessen by the time pressure situation even it appears a slightly rise between Trials 6 and 7 .

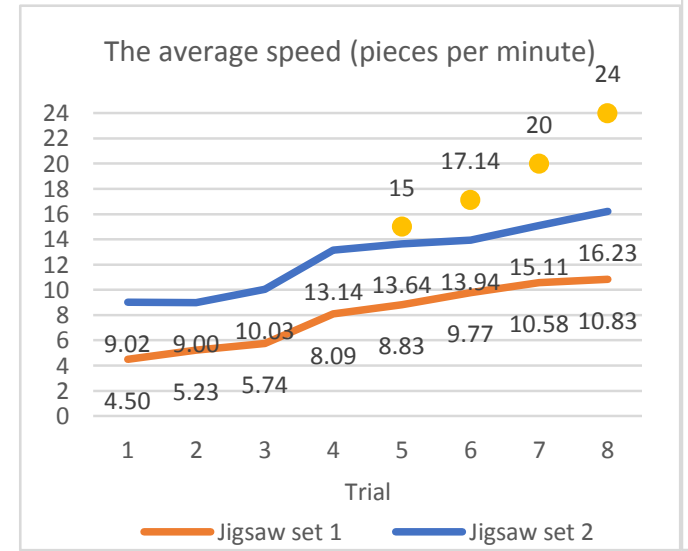

(a)

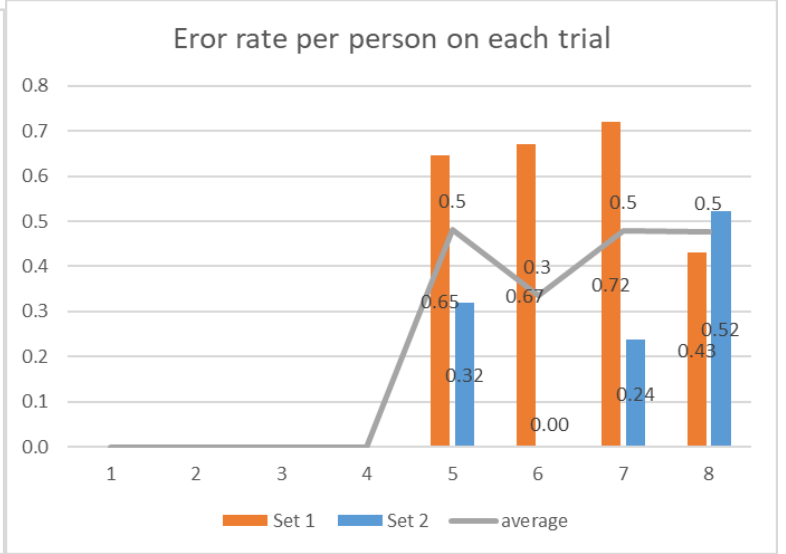

(b)

Figure 5. The speed and accuracy outcome for Task 2



(a)

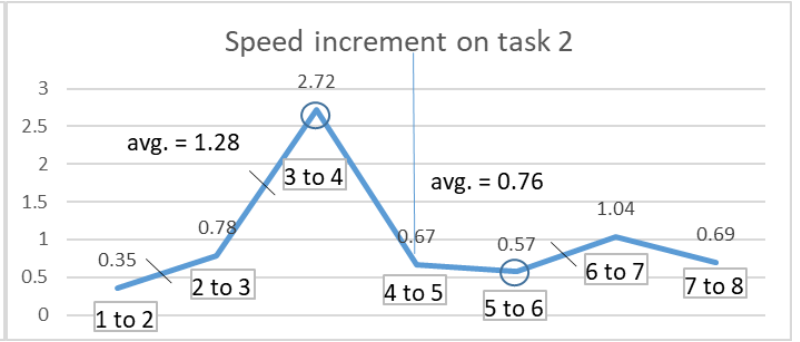

.... (b)

Figure 6. The average speed increment on Task 1 and Task 2

Although the speed increment was all positive on Task 2, it was found that the speed increment on Task 1 , appears to be higher than on Task 2 . The probable reason might be the characteristic of task. Task 1 was a discrete process involving a short sequence that was independent from each other. In other words, it engaged the amount of working memory less than the jigsaw task. The jigsaw task was more difficult as the interface involved spatial information of the moving pieces and every piece needed to be integrated. Therefore, participants tended to get extra pressure and require a higher degree of mental energy which directly affected brain fatigue, then impacted the task performance. This implied that the performance on a fixed interface might be more robust to the effect of psychological disturbance.

On the other hand, Figure 5(b) presents the error rate that occurred during the time pressure condition. The number of errors was not available in the normal session because errors that occurred in the process were corrected by participants before the task completion. However, the number of errors appears to increase. The average error was 0.4 pieces per trial. The number of errors seems to drop in Trial 6 as the participants adapted themselves to the circumstance and tried to prevent the previous error. However, it appears to rise again on Trial 7 and 8 . Overall, the error rate tended to increase by the level of stress.

\subsection{Error pattern}

Task1 The mistake patterns were categorised into 7 types (Figure 7). While Figure 8 shows some examples of error characteristics consisting of pressing the wrong button (a), pressing incorrect order (b), problems with the shift key (c), switching to another word (d), typing excess letters (e) and missing some keys (f). Participants perhaps misinterpreted the symbol, confused the appropriate key series or loss of precision on the position of the button. These errors often occurred repeatedly or in the same manner. Numerous errors have recurred before it was aware and prevented later. Zeidner and Matthews (2011) claimed that pressure and anxiety could deteriorate thoughts and consciousness. Thus, even participants did their best, the error still occurred. 


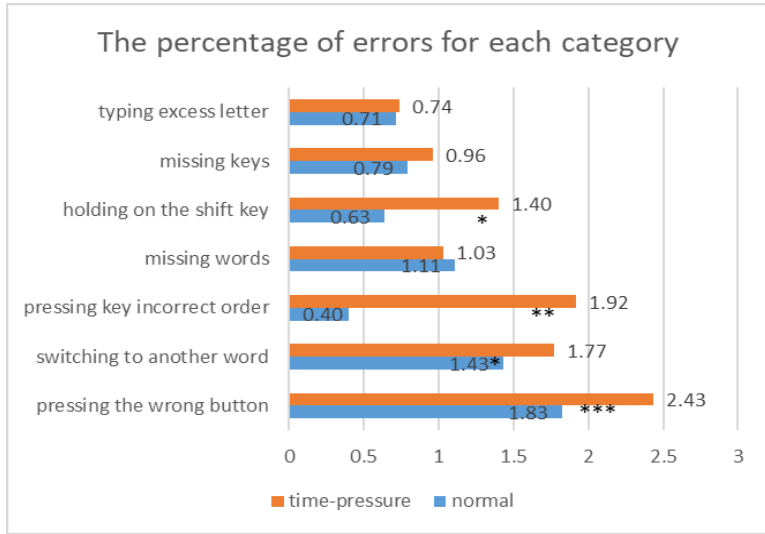

(a)
The proportion of errors categorised by the word pattern
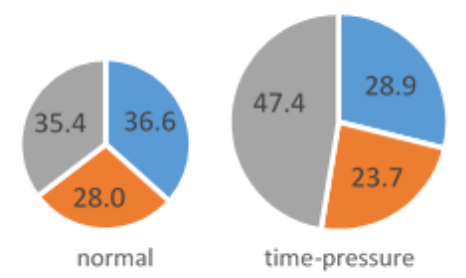

- $\mathrm{Fi}$

- pvf

- ivp

(b)

Figure 7. The proportion of erroneous cause and the frequent error words

Overall, most errors significantly increased in the time-pressure situation with exception to the problem of the missing word which decreased slightly. It was possible that participants might pay much attention to the screen in a stressful situation, so they miss some words not as much. However, the data showed that pressing incorrect sequence, pressing the wrong button and dealing with the shift key, and switching to another word, increased significantly in a stressful situation. The causes were from a finger placement, the key sequence, word recognition as well as unsynchronised action on controlling keys. Participants sometimes could not run a cognitive process, interact on the interface and aware of mistakes properly in a timely manner, like an action slip, according to Norman (1984) paper. That is they might perform under their subconscious and familiarity.

The interface on which participants saw had a different letter to the letter on the screen. They needed to decode and press the letter in the correct sequence. Most errors were from buttons in close vicinity (Figure 8(a)). Sometimes participants miss the button series. It was found that the frequent errors are from the pattern of 'ivp' followed by 'Fi' and 'pvf', respectively (Figure 7(b)). From the investigation, the probable reason might be the interaction on 'ivp' quite swing from left-right-left and the button position did not link onto the next button cue (Figure 3). While the step on typing 'Fi' and 'pvf' was rather smooth, consistent and linkage. In other words, the distance between the buttons, and the flow of these two words were clear and not too transitional. 'Fi', another popular error word often caused by controlling the shift key. Participants often forgot to press or hold on the shift key. This implied that concurrent action on the keyboard tends to raise a mistake.

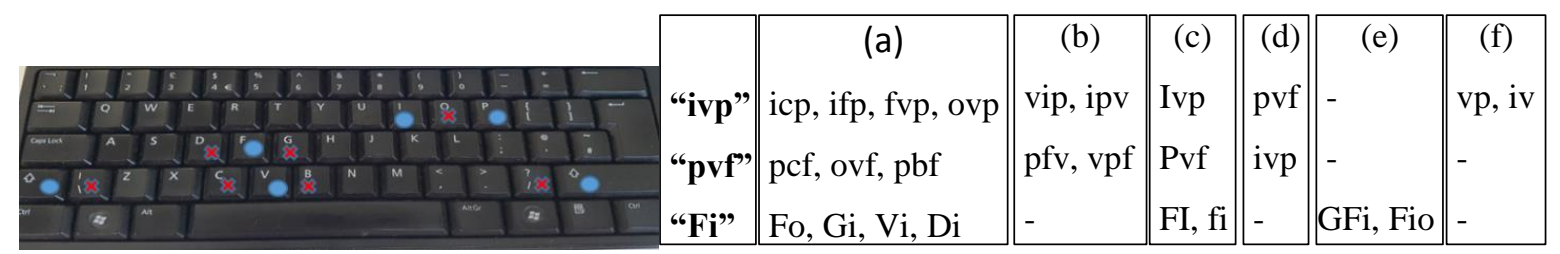

Figure 8. The characteristics of error on Task 1 (decoded from Thai to English letter)

Task 2 The error patterns were divided into 2 types: wrong piece (the most, 61.5 percent) and wrong direction as shown in Figure 9. In terms of spatial layout, the wrong piece meant putting a piece in the wrong position. The secondary error was putting a piece in the wrong direction (rotation problem). Due to the jigsaw operation is direct manipulation, participants perceived their feedback on time. The mistake must be corrected otherwise the pieces cannot be connected. When participants were overloaded with information and time pressure, they usually cannot see the little difference between pieces bring about selecting the wrong piece. In addition, the piece of jigsaw seems symmetry which has two outs at each end and two dents in the middle. Participants can rotate 180 degrees for the horizontal piece to find the correct direction. If they turn the horizontal 90 degrees to the vertical or turn the vertical 90 degrees to the horizontal, it cannot fit into the nearby piece as the same type would not piece together. In a difficult situation, participants tended to lose the capacity of semantic reasoning and forgot the image relationship between the pieces. 


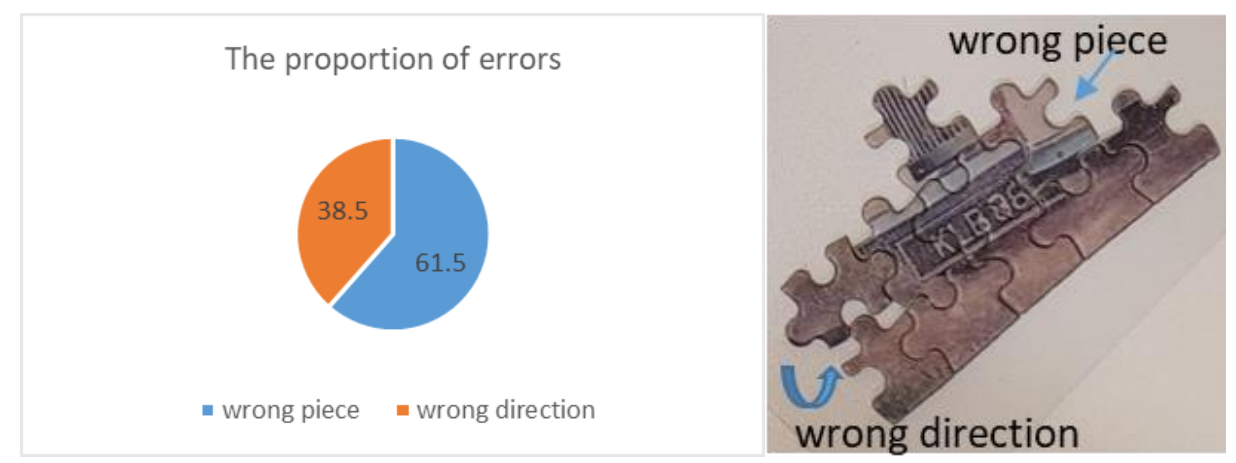

Figure 9. The proportion and the characteristics of error on Task 2

\subsection{Behaviour and strategy}

Apart from the task performance, it can be seen that participants used various strategies and movements. They intended to concentrate on the works, tried to memorise data in a short time, retrieve the information and perform tasks to their best ability. Many physiological responses from participants probably alleviate stress both directly and indirectly during the test. For example, sighing, deep breathing, touching on their head, and wagging or taping their finger on the table. Even their heart rate also widely fluctuated and increased during the experiment.

Task1 For the command 'Fi', most of the participants often used the left little finger to press the shift button along with pushing the ' $\mathrm{f}$ ' letter key by the left index finger and used another index finger to press the 'i' in the right as a regular basis (Figure 10(a)). Nevertheless, someone decided to use the left thumb to press the shift and the right middle finger to press the 'f' letter before moving that finger to the 'i' button on the right side (Figure 10(b)). While somebody tended to use the shift button on the right with a little finger and use the left index finger to press the letter at the same time shown in Figure 10(c) providing reason that it could better for hand mobility synchronization. Surprisingly, two participants decided to use the caps lock button instead even this need two keystrokes. They claimed that pressing the shift and character buttons simultaneously may easily slip. When performing in the time constraint situation, it can be seen that many participants tend to change finger strategies which the aim of boosting the operation performance. For example, implicitly changed from using the middle finger to the index finger or using the index finger of the right hand to press the letter on the left side of the keyboard. In sum, it suggested that the cognitive workload influence on changing behaviour and strategy on the task.

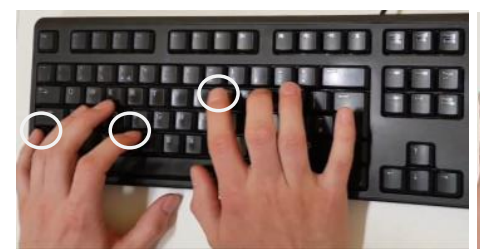

(a)

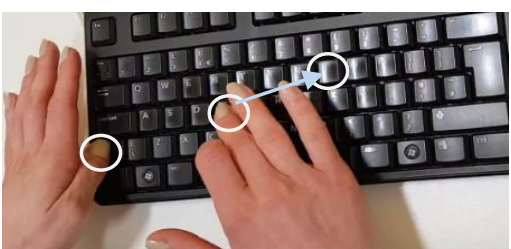

(b



(c)

Figure 10. The various finger postures on keyboard

Task2 Ehrlich (1996) claimed that a person who has good visual skill can create a better mental model for information navigation. It was found that most participants started forming a picture with the base row by finding the bottom-edge pieces first, then judging and putting them into place. After that, they sorted the inside pieces that were looked similar, clustered them, or sought the target piece to build up part from the base row as seen in Figure 11. Under the time-limited conditions, they often were not able to find the piece properly, fit pieces together effectively and many actions seemed slower. They seemed to lose the accuracy of finger movement. Many participants often fitted the piece into place correctly but finally, removed it. In other words, responses in hand control were imprecise by mental overload. Their confidence in the task also lessened. The researchers observed that they took a lot of time for rotating the piece or tried to compress into the wrong place. Indeed, participants failed to organise semantic information properly. Most 
participants could remember around at least 8 pieces from the whole picture. They often assembled these pieces first and then seemed slower in connecting the additional pieces. Although participants have planned before, sometimes they have to solve a problem at prompt. If they cannot find the target piece, they will select any piece and tend to trial and error on that piece. Somebody said that just believe his/her instinct according to Illera et al. (2010). According to Norman (1983), the mental model was often incomplete and unscientific. In the high-stress condition, most participants cannot develop an eye with fine inspection on each piece or even consider the shape of the piece for rotation. Richardson and Vecchi (2002) suggested that the limited working memory capacity impact on cognitive performance.


Figure 11. The common strategy used in Task 2

\section{General discussion}

The mental pressure could affect physical processes leading to a mistake and a decrease of effectiveness. The interface types and task complexity affected differently to task performance. Similar findings were found by Wu et al. (2016) and Hughes and Babski-Reeves (2005). Moreover, this study suggested that the fixed interface might be more robust to the psychological disturbance than the moving interface.

The time pressure influences cognition, recognition, judgment and fine motor control of operators. Illera et al. (2010) suggested that the design solution should consider both the quantitative criteria and qualities of composition supporting the cognitive ability. Some recommendations to accommodate user behaviour in high-pressure situation based on this findings are the following:

The fixed interface The interface layout should be appropriate and the adequate space or distance between each key should be considered. The sequence of tasks or buttons should be smooth in the process of transition. To accomplish the task with dexterity, the control panel should be designed for performing in a balanced manner or synchronising mobility. To prevent consecutive errors, the feedback should be immediately provided to the users such as graphics highlighted or audio. It should be suggested that if users are able to balance their attention equally on both hands and assign a finger on a specific button in the working area, they will operate at a higher rate with a spatial map they generated. Moreover, the steps should be transferred from the same direction, providing the consistency link to the next button. The interface should be easy to recognize and should not much burden the user's memory.

The moving interface Designers and engineers should decrease the diversity of interface patterns by grouping or naming them suitably, and facilitate the orientation or navigation to users. For example, give a clue to easily arrange so the number of rotations will be reduced. If the part is symmetry in one axis, it will have two options for orientation. On the other hand, if it is asymmetry, it will be easier to recognise and align. It could be beneficial in providing a clear image with the outstanding colour, form an observing point or other non-spatial attribute information. If the interface offers an explicit relationship between the sections and the spatial reference, users will develop a mental model effectively. Finally, a confirmation or a suggestion should be given to enhance the user's confidence.

The findings also suggested that the stress could heighten a person's ability as seen from various strategies participants used or adapted. These implied that participants can learn and interact better with the fixed interface than the moving interface under difficult conditions. The empirical study on interface and interaction provided much understanding that interface design can impact the speed and accuracy of the task. If practitioners design it effectively, it can reduce error, enhance the users' skill and the flow experience (Csikszentmihalyi, 1997), or even the peripheral interaction (Bakker et al., 2016; Probst, 2016). Thus, it sounds promising for seeking design intervention by the appropriate interface to mitigate the user's mistake in the practical situations that are full of stress and complexity as Rasmussen and Vicente (1989) suggested. 


\section{Conclusion}

This experimental study explores cognitive behaviour and performance accuracy for the dexterous operations on the fixed and moving interface between normal and stressful situations. The findings indicated that the time-stress brings about a reduction in task performance. Although the efficiency (speed) was increased, the effectiveness and accuracy were degraded. The results also implied that the performance on a fixed interface might be robust in that participants can learn and interact better under stressful condition. The exploration from the experiment brings about insights about the cognitive ergonomics, the interaction style, the locus and the nature of errors. This will be a knowledge base and motivation for future research.

\section{References}

Bakker, S. et al. (2016), "Introduction: Framing Peripheral Interaction”, In: Bakker, S. (Ed.), Peripheral Interaction, Human-Computer Interaction Series, Springer, Switzerland, pp. 1-10. https://doi.org/10.1007/ 978-3-319-29523-7_1

Csikszentmihalyi, M. (1997), Finding flow: the psychology of engagement with everyday life, Basic Books, New York.

Ehrlich, K. (1996), “Applied mental models in human-computer interaction”, In: Johnson-Laird, P.N., Oakhill, J. and Garnham, A. (Eds.), Mental models in cognitive science, Psychology Press, East Sussex, pp. $223-245$.

Hong, J.C. et al. (2012), "Effects of cognitive style on digital jigsaw puzzle performance: A GridWare analysis", Computers in Human Behavior, Vol. 28 No. 3, pp. 920-928. https://doi.org/10.1016/j.chb.2011.12.012

Hughes, L.E. and Babski-Reeves, K. (2005), "Time pressure and mental workload effects on perceived workload and key strike force during typing”, Proceedings of the Human Factors and Ergonomics Society Annual Meeting, SAGE, Los Angeles, CA, pp. 1390-1394. https://doi.org/10.1177/154193120504901427

Illera, C. et al. (2010), "NO_PANIC. "Escape and Panic in Buildings"-Architectural Basic Research in the Context of Security and Safety Research", In: Klingsch, W., Rogsch, C., Schadschneider, A. and Schreckenberg, M. (Eds.), Pedestrian and Evacuation Dynamics 2008, Springer, Berlin, Heidelberg, pp. 733-742. https://doi.org/10.1007/978-3-642-04504-2_71

Norman, D.A. (1983), "Some observations on mental models", In: Gentner, D. and Stevens, A. (Eds.), Mental models, Lawrence Erlbaum Associates, New Jersey, pp. 7-14.

Norman, D.A. (1984), "Stages and levels in human-machine interaction”, International Journal of Man-Machine Studies, Vol. 21 No. 4, pp. 365-375. https://doi.org/10.1016/S0020-7373(84)80054-1

Probst, K. et al. (2016), "Peripheral interaction in desktop computing: why it's worth stepping beyond traditional mouse and keyboard", In: Bakker, S. (Ed.), Peripheral Interaction, Human-Computer Interaction Series, Springer, Switzerland, pp. 183-205. https://doi.org/10.1007/978-3-319-29523-7_9

Proctor, R.W. and Vu, K.L. (2016), "Principles for Designing Interfaces Compatible with Human Information Processing”, International Journal of Human-Computer Interaction, Vol. 32 No. 1, pp. 2-22. https://doi.org/ 10.1080/10447318.2016.1105009

Rasmussen, J. and Vicente, K.J. (1989), "Coping with human errors through system design: implications for ecological interface design”, International Journal of Man-Machine Studies, Vol. 31 No. 5, pp. 517-534. https://doi.org/10.1016/0020-7373(89)90014-X

Richardson, J.T. and Vecchi, T. (2002), “A jigsaw-puzzle imagery task for assessing active visuospatial processes in old and young people", Behavior Research Methods, Instruments, \& Computers: A Journal of the Psychonomic Society, Vol. 34 No. 1, pp. 69-82.

$\mathrm{Wu}$, L. et al. (2016), "Influence of information overload on operator's user experience of human-machine interface in LED manufacturing systems", Cognition, Technology \& Work, Vol. 18 No. 1, pp. 161-173. https://doi.org/10.1007/s10111-015-0352-0

Young, M.S. et al. (2015), "State of science: mental workload in ergonomics”, Ergonomics, Vol. 58 No. 1, pp. 117. https://doi.org/10.1080/00140139.2014.956151

Young, R.M. (1983), "Surrogates and Mappings: Two kinds of conceptual models for interactive devices", In: Gentner, D. and Stevens, A. (Eds.), Mental models, Lawrence Erlbaum Associates, New Jersey, pp. 35-52.

Zeidner, M. and Matthews, G. (2011), Anxiety 101, Springer Pub. Co, New York. 